\title{
Editorial: Sustainable Mobile Networks and its Applications
}

\author{
A. Pasumpon Pandian ${ }^{1}$. Joy long-Zong Chen ${ }^{2} \cdot$ Zubair A. Baig $^{3}$
}

Published online: 19 March 2019

(C) Springer Science+Business Media, LLC, part of Springer Nature 2019

\section{Editorial:}

Mobile networks have a large scale of potential applications like smart mobile computing, cloud computing, secure communication, cyber physical systems, multimedia communication and Internet of things environment. Development of sustainable technologies in mobile networks meet the demands and requirements of its applications. Sustainable mobile networks aiming in the reduction of energy consumption and improving the performance and efficiency of the networks. Many network technologies involved and gained the tremendous achievements in sustainable mobile networks and that includes cognitive radio networks, vehicular ad hoc networks, sensor networks, neural networks, routing strategies, detection of malicious attacks and secure routing algorithms. The goal of this special issue is to bring the theoretical preparations and practical applications of research contribution in all aspects of sustainable mobile networks and applications.

This special issue features 14 selected papers with high quality. The first article "Big Data Oriented Energy Aware Routing for Wireless Sensor Networks" achieved efficient routing data load distribution by selecting multiple low cost paths and involving these paths for data packet routing. The empirical results demonstrate orders of magnitude load distribution effectiveness and slightly increased total energy consumption of the proposed routing technique when compared to least energy cost routing protocol.

\footnotetext{
A. Pasumpon Pandian

pasumponpandian32@gmail.com

Joy Iong-Zong Chen

jchen@mail.dyu.edu.tw

Zubair A. Baig

z.baig@ecu.edu.au

KGiSL Institute of Technology, Coimbatore, India

2 Department of Electrical Engineering, Dayeh University, Dacun, Taiwan

3 Edith Cowan University, Joondalup, Western Australia
}

The second article titled "A Novel Algorithm for Enhancement of Energy Efficient Zone Based Routing Protocol for MANET" deals with a novel algorithm to increase the energy efficient zone based routing protocols which control the network topology by estimating node die out rate. Furthermore, a game theory approach with energy efficient zone based routing protocol to improve QoS routing for MANET. Finally, the experimental outcomes proved the efficiency of the proposed algorithm compared with other routing algorithms. In the next article with the title of "Secure P2P Intelligent Network Transaction using Litecoin" authored by Padmavathi presents the online transaction from one person to another is studied in peer to peer networks with reduced transaction time. Here, a block chain distribution in bit-torrent network is considered to transact the litecoins. Finally, efforts are considered to avoid double spending attacks on litecoins.

The fourth article entitled "Group Based Multi-Channel Synchronized Spectrum Sensing in Cognitive Radio Network with $5 \mathrm{G}$ ". This paper investigates the problem of Cognitive Radio Network (CRN) with Cooperative Spectrum Sensing (CSS), when multiple idle channels are available. To enlarge network connectivity and provide larger coverage for users, author integrate CRN-CSS with $5 \mathrm{G}$. Trinary partitioning is performed to group user and perform sensing in cooperative manner. Sensing of multiple channels leads to interference that is overwhelmed by the novel Dynamic Multi-Channel Slot Allocation (DMCSA) algorithm which allocates channel effectively. Nano-grids are a replication of large electricity grids resembling their model but only on a smaller scale to locations that are not accessible easily and the connectivity of grid is not possible. Fifth article titled "Cost optimization of PV-Diesel Systems in Nanogrid Using L J Cuckoo Search and its Application in Mobile Towers". Generally, these are used in remote hilly regions where there can be a renewable source of energy. For the purpose of standalone nano-grid, an algorithm which is simple to determine the required Photo Voltaic (PV) panel, a Diesel Generator (DG) along with battery models are used.

The sixth article titled "Wavelength Allotment for All-toAll Broadcast in WDM Optical Modified Linear Array for 
Reliable Communication" authored by Sabrigiriraj. In this paper, a linear array network is modified by additionally joining alternate nodes. For such a modified linear array, the minimum wavelength number needed to support all-to-all broadcast is derived by grouping non overlapping connections on a common wavelength. The results obtained for modified linear array shows that the wavelength number needed is approximately reduced by half when compared with that of a linear array. Next article titled "Spectrum Prediction in Cognitive Radio with Hybrid Optimized Neural Network" presents a new class of optimization heuristics called hybrid optimization is used. This will implement two or more algorithms for the same optimization. A Genetic Algorithm along with Particle Swarm Optimization (GAPSO) method is proposed with a Back-Propagation Neural Network (BPNN) as a novel supervised learning algorithm for predicting spectrum patterns in cognitive radio networks. Based on this concept these open systems enabling innovation in new services are particularly important for funded IoT infrastructure in order to maximize the benefit for the society.

The Eighth article entitled "A Novel Research on the Influence of Enterprise Culture on Internal Control in big Data and Internet of Things". This research work proposes a novel technique for pattern recognition incorporating group clustering and convex optimization process for reducing the data size of the feature set extracted from the clusters in the specific group and optimization to obtain a minimal value of error convergence in the least possible time. Next article titled "Particle Swarm Based Resource Optimized Geographic Routing for Improved Network Lifetime in MANET" presented by Nallusamy. In order to improve the resource optimization and network lifetime, an efficient Particle Swarm based Resource Optimized Geographic Routing (PS-ROGR) technique is introduced in MANET. Initially, each particle (i.e. mobile node) movement in a network is controlled by its local best known position in the search space (i.e. geographic location). The PSO permits all the particles in the network to communicate with the other particles with minimum energy.

The tenth article proposed the fuzzy logic strategy to detect black hole attack based on certificate authority, energy auditing, packet veracity check and trust node to improve the performance of AODV. Fuzzy schema is a mathematical logic that attempts to work out problems by assigning the prediction values to an imprecise range of data. Fuzzy logic detect misbehaving node by giving certificate to only trusted node. The proposed technique is more secure and reliable in military data communication. Next article entitled "Development of Fuzzy based Energy Efficient Cluster Routing Protocol to Increase the Lifetime of Wireless Sensor Networks". Balaji proposed a multi hop transmission, where the data packets are send from one hop to another hop. Finally these data packets are transmitted to the base station. To transmit the packets from source sensor to wireless sensor network base station via the cluster head, using the fuzzy logic type 1with three parameters such as trust factor and distance.

Next article entitled "Influence of Krylov subspace in Graph Isomorphism for Mobile Networks" represent the graphs in a common mathematical space (Symmetric Positive SemiDefinite space), so that two isomorphic graphs always map to the same coordinates in a mathematical space. Experiments are conducted using publicly available benchmark graph database. From the simulation, it is observed that the representation recommended in this work acts like a signature for each graph with guaranteed isomorphism. The thirteenth article titled "Target Aucourant Data Dissemination by Collaboratory Sensing in IOT Environment" presents an efficient Event Based Echeloned Topology (EBET) is deployed using convex hull for electing collaborators and typically constructing event based region for the coordinator nodes using $\mathrm{R}+$ tree. Thus, the proposed topology with efficient intra and inter aggregation reduces overall latency, energy consumption and also preserved the data accuracy of the nodes thereby network lifetime is improved.

The last article entitled "Efficient Secure Aggregation in VANETs Using Fully Homomorphic Encryption (FHE)" improves the privacy of nodes in vehicular networks with reduced communication overhead. The performance of the proposed FHE is compared with Paillier cryptosystem. It is found that the proposed method provides less communication overhead to transmit the message with improved security than with Paillier cryptosystem.

Acknowledgements The guest editors are thankful to our reviewers for their effort in reviewing the manuscripts. We also thank the Edit-in-Chief, Dr. Imrich Chlamtac for his supportive guidance during the entire process.

Publisher's Note Springer Nature remains neutral with regard to jurisdictional claims in published maps and institutional affiliations.

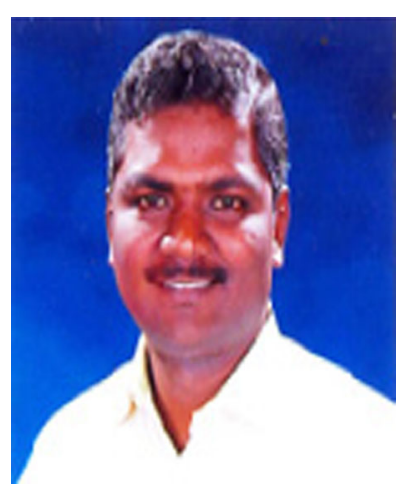

Dr. A. Pasumpon Pandian has received his Ph.D. degree in the Faculty of Information and Communication Engineering under Anna University, Chennai, $\mathrm{TN}$, India in 2013. He received his graduation and postgraduation degree in Computer Science and Engineering from PSG College of Technology, Coimbatore, TN, India in the year 1993 and 2006 respectively. He is currently working as Professor in the Computer Science and Engineering department of KGiSL Institute of Technology, Coimbatore, TN, India He has 26 years of experience in Teaching, Research and IT industry. He has published six research articles in journals. His research interest includes, Image Processing and Coding, Image Fusion, Soft Computing and Swarm Intelligence. 


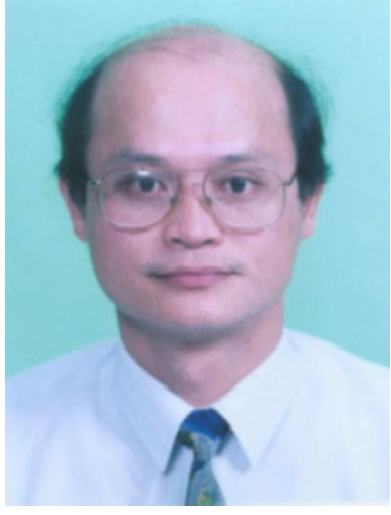

Dr. Joy long-Zong Chen is currently a full professor of Department of Electrical Engineering Dayeh University at Changhua Taiwan. Prior to joining the Dayeh University, he worked at the Control Data Company (Taiwan) as a technical manger since Sep. 1985 to Sep. 1996. His research interests include wireless communications, spread spectrum technical, OFDM systems, and wireless sensor networks. He has published a large number of SCI Journal papers in the issues addressed physical layer for wireless communication systems. Moreover, he also majors in developing some applications of the IOT (Internet of Thing) techniques and Dr. Joy I.-Z. Chen owned some patents authorized by the Taiwan Intellectual Property Office (TIPO).

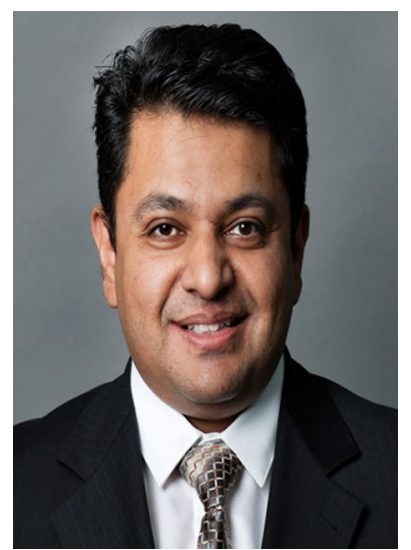

Dr. Zubair A. Baig received the B.S. degree in computer engineering from the King Fahd University of Petroleum \& Minerals, Dhahran, Saudi Arabia, in 2002, M.S. degree in Electrical Engineering from the University of Maryland, College Park, USA, in 2003 and the Ph.D. degree in computer science from Monash University, Melbourne, Vic., Australia, in 2008. Currently, he is a Senior Lecturer in Cyber-Security, Edith Cowan University, Western Australia. He has authored over 70 journal and conference articles and book chapters. His research interests are in the areas of cyber-security, artificial intelligence, Internet of Things, and optimization algorithms. He is serving as the editor of the IET Wireless Sensor Systems Journal and the PSU - A Review Journal, Emerald Publishing House. He has served on numerous technical program committees of international conferences and has delivered more than 12 keynote talks on cyber security. 